\title{
Monopulse angle estimation with MIMO radar
}

\author{
Aleksandr N. Semenov ${ }^{1, *}$, Vladimir I. Krainy ${ }^{1}$, and Ivan A. Rodichev ${ }^{1}$ \\ ${ }^{1}$ Bauman Moscow State University, Radio electronics and Laser Technic Department, 105005 2-ya \\ Baumanskaya ul. 5, Moscow, Russia
}

\begin{abstract}
In this article a generalization of the amplitude monopulse method for angle estimation for radars with multiple input and multiple output (MIMO) elements is discussed. Bearing characteristics for sparse virtual antenna arrays are presented. A reconstructed image of the radiation source is presented for two cases of direction finding: based on calculation of the correlation integral and based on weighted sum of signals over the aperture of the virtual antenna array.
\end{abstract}

\section{Introduction}

The research of unmanned transport control is undergoing explosive development. In conditions of poor visibility of the environment, the most high-quality solution in the problem of observing and tracking obstacles is obtained by radars. To ensure high angular resolution and accuracy, it is necessary to increase the size of the antenna system on the one hand and, on the other, move to shorter wavelengths [1]. These will lead to more complicated overall system and increase it total cost. The alternative to obtaining accurate angular estimates of targets is monopulse systems that combine simplicity of implementation and low computational complexity.

The concept of combining radar systems with multiple input and multiple output elements (MIMO) and ordinary monopulse systems is described in the literature [1-3]. In this study, we consider the use of a sparse MIMO system for accurate estimation of the angular position of the target, in which the positions of the receiving and transmitting elements coincide, and the elements themselves can change their functional purpose from the receiver to the transmitter via a microwave switch.

\section{MIMO system model}

Consider a radar system consisting of a linear antenna array with a total number of switched elements $n$. Each element can operate either in signal transmission mode or in receiving mode, providing at the same time one transmitting and $N_{r}=n-1$ receiving positions. The receiving positions will determine the receiving antenna array. The total number of positions of the transmitting elements is $N_{t}=n$ positions that will form the transmitting antenna array.

Suppose the target located in the far field zone for each of the elements of the antenna system, then the front of the reflected wave will be flat, and thus, the delays $\mathbf{A}(\theta)$ (steering

\footnotetext{
* Corresponding author: semenov.an@,bmstu.ru
} 
vector) of the reflected signals will sequentially increase in the elements of the receiving array including the propagation time lag from the current transmitting element to the target.

Each receiving element of the antenna array receives signals from all positions of the transmitting elements, except for those in which the transmitting element is currently located. Thus, the total number of received signals will be $N=N_{t} \cdot N_{r}$, which can be considered as received by a virtual antenna array of $N$ elements [1-3].

It is known that the directivity pattern $F(\varphi)$ of a linear antenna array can be determined from the following equation:

$$
F(\varphi)=\mathbf{w}^{\mathrm{T}} \mathbf{A}(\varphi)
$$

where $\mathbf{w}$ is the vector of amplitudes in the antenna elements and $\mathbf{A}(\varphi)$ is the steering vector, $\varphi$ is direction.

The virtual antenna directivity pattern can be calculated the same way as in equation (1) replacing $\mathbf{A}(\varphi)$ to $\mathbf{A}(\theta)$, the vector of delays in received signals produced for all combinations of transmitted positions and received positions. To avoid grating lobes in the beam pattern of virtual steering vectors, the distance between two virtual elements must be no larger than $\lambda / 2$, which requires the proper antenna configuration.

The received MIMO signal model is expressed as:

$$
\mathbf{Y}(t)=\beta \mathbf{A}(\theta) s(t-\tau)+n(t)
$$

where $\beta$ is the amplitude of target source, $\mathbf{A}(\theta)$ is the steering vector of the virtual antenna array, $s(t-\tau)$ is the received signal and $n(t)$ is the noise assumed to be spatially independent.

Using proper $\mathbf{w}$ can lead sum and difference directivity pattern with known properties [1] that can be used in monopulse technique for target angular position estimation. The (1) and (2) equation present independent time and spatial signal processing. The more effect can be yield by combined processing expressed in complex correlation integral:

$$
Q\left(\mathbf{r}, \mathbf{r}_{0}\right)=\int \mathbf{Y}(t) \cdot \mathbf{Y}_{0}^{*}(t) d t
$$

where $\mathbf{r}$ is radius vector to the target position and $\mathbf{r}_{\mathbf{0}}$ is radius vector to the reference target position, (.)* is complex conjugated symbol.

Substituting equation (2) into (3) yield expression:

$$
Q\left(\theta_{0}, \tau_{0}\right)=\mathbf{A}^{T}\left(\theta_{0}, \tau_{0}\right) \int \mathbf{Y}(t) \cdot s^{*}\left(t-\tau_{0}\right) d t=\mathbf{A}^{T}\left(\theta_{0}, \tau_{0}\right) \mathbf{u}(t),
$$

where $\mathbf{u}(t)$ is the convolution of input signal on virtual antenna array with reference signal $s\left(t-\tau_{0}\right)$.

The equation (4) structure is like equation (1). The difference between (1) and (4) is a way how the beam steering is made. The (1) equation need a calculation of $\mathbf{w}$ vector, then the maximum signal is appeared on angle where target is located. The equation (2) need a calculation of $\mathbf{A}\left(\theta_{0}\right)$ for all possible target angular positions $\theta_{0}$, then the maximum signal is one of all observed angles which the target can has. The advance of using equation (4) is that it can be used with sparse antenna arrays without angle ambiguity.

\section{Monopulse angle estimation in sparse MIMO radar}

Amplitude monopulse angle estimation is based on angular error calculation produced by amplitude ratio between signals from sum and difference antenna pattern. The resulted angular target position is calculated by sum antenna direction and calculated error as shown in expression: 


$$
\hat{\theta}=\theta_{a n t}+r^{-1} \cdot \operatorname{Im}\left\{\frac{\mathbf{w}_{\Delta}^{T} \mathbf{Y}(t)}{\mathbf{w}_{\Sigma}^{T} \mathbf{Y}(t)}\right\},
$$

where $\theta_{\text {ant }}$ is current antenna direction, $\mathbf{w}_{\Delta}$ is difference weighing coefficients for the partitial direction pattern, $\mathbf{w}_{\Sigma}$ is sum weighing coefficients for the partitial direction pattern, $\mathbf{Y}(t)$ is MIMO signal, $r^{-1}$ is inverse function to the bearing characteristics of monopulse method expressed as:

$$
r=\operatorname{Im}\left\{\frac{\mathbf{w}_{\Delta}^{T} \mathbf{A}(\theta)}{\mathbf{w}_{\Sigma}^{T} \mathbf{A}(\theta)}\right\},
$$

where $\mathbf{A}(\theta)$ is steering vector of virtual antenna.

Substituting expression (4) in (5) and (6) the bearing characteristics for the MIMO system based on complex correlation integral were get. It was compared with the characteristics obtained through the weight functions [1]. Comparison of bearing characteristics were made on virtual antenna array of $N=56$ elements obtained by changing the position of the transmitter at $n=8$ positions of the receivers. The step between the elements is $2 \lambda$, the working wavelength is $\lambda=0.125 \mathrm{~m}$. The target located at $50 \mathrm{~m}$ and the observable sector has bounds from $-45^{\circ}$ to $+45^{\circ}$.

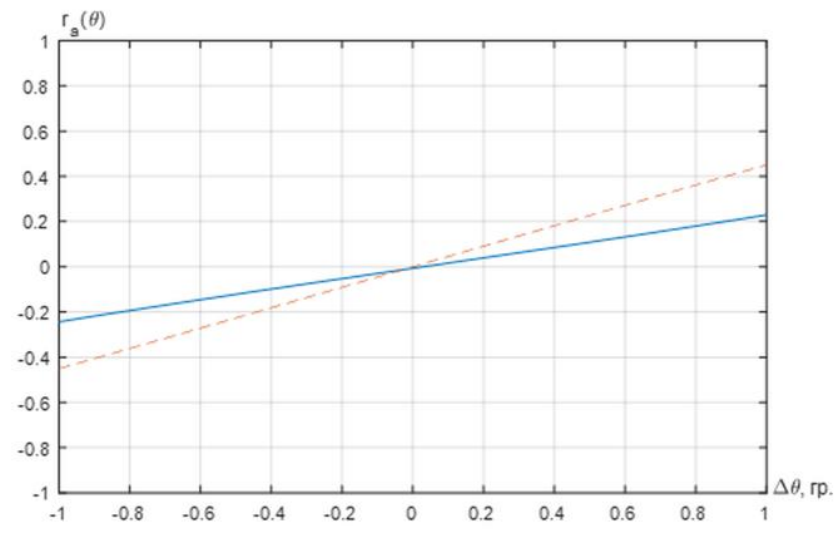

Fig. 1. Bearing characteristics calculated by weights (dashed line) and by complex correlation integral (solid line).

It can be seen from Fig. 1 that the bearing characteristics are numerically close to each other and are characterized by enough linearity. Fig. 2 shows the reconstructed images of the target signal in space for a sparse MIMO system and two direction finding methods: through the complex correlation integral a) and through the weight functions b). With comparable bearing accuracy, the proposed method allows to suppress secondary main lobes, and therefore, does not have ambiguity in estimating the angular position.

\section{Conclusion}

It is shown that the calculation of the complex correlation integral in a spatially multichannel MIMO system can be considered from the point of view of the diagram pattern of a virtual antenna array with a known phase shift vector that is a linear combination of phase shifts of the signal propagating from the transmitter to the target and from the target to the receiving element. The target angular position estimation obtained by calculating the complex correlation integral is not ambiguous and is characterized by high accuracy. 


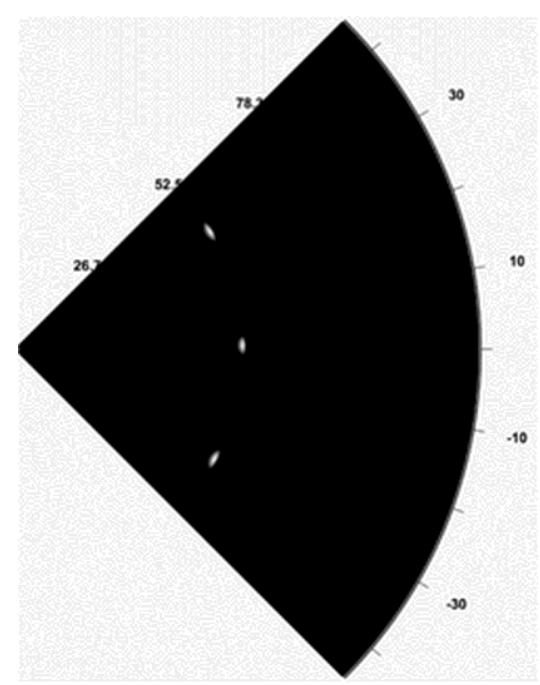

a)

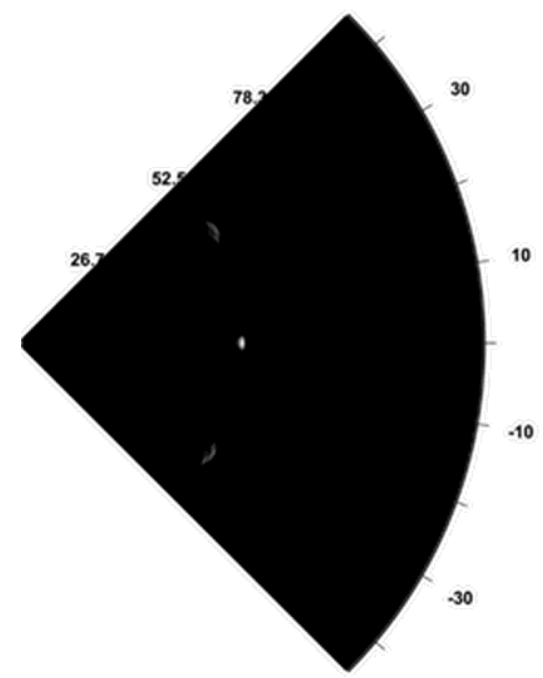

b)

Fig. 2. The reconstructed images of the target signal with a) weighted coefficients and b) with complex correlation integral.

\section{References}

1. R. Feng, F. Uysal, P. Aubry, A. Yarovoy. Sonar Navigation IET Radar. 12(10). p. 1131-1136 (2018)

2. V.V. Chapurskiy. Vestnik MGTU im. N. E. Baumana, Priborostroenie. 4, p. 72-91 (2011)

3. V.I. Krainy, A.N. Semenov. Vestnik MGTU im. N. E. Baumana, Priborostroenie. 6, p. $31-40(2016)$ 